\title{
Self Propagating High Temperature syntheses and gas sensing properties of BiFeO3 thin films
}

\author{
Martirosyan Norayr \\ State Engineering University of Armenia \\ Microelectronics and Biomedical Devices Department of SEUA \\ Teryan 105, 0009, Yerevan, Armenia. \\ E-mail: marnorw@yahoo.com
}

\section{Introduction}

Current interest in perovskite and perovskite-related oxides is based on a wide spectrum of their electrical, dielectric, acoustic and magnetic properties. Due to presence of oxygen vacancies and ionic conductance, perovskite oxides have a high catalytic activity to oxygen reduction and oxidation, and thus suitable for a large variety sensor applications. A group of perovskites known as multiferroics offer a rich variety of physical properties making them the most multifunctional materials. In particular the cross coupling between the dielectric/electrical, magnetic and acoustic properties makes it possible to develop new components with radically new functionalities for applications in sensors, microwave and $\mathrm{THz}$ devices etc. $\mathrm{BiFeO}_{3}$ is one of the most studied multiferroics considered for these applications.

Special attention is paid to acetone, ethanol, natural gas and petrol hazardous gas sensing properties of $\mathrm{BiFe}_{1-x} \mathrm{Mn}_{\mathrm{x}} \mathrm{O}_{3}$. Because of rapid and accurate identification of hazardous situations to detect and warn of an occurrence of a chemical incident within enclosed spaces, such as buildings and transportation facilities is of great importance. These gases are associated with fires and mining operations, and it is of highest importance to warn and protect operators from potential harm caused by overexposure to high concentrations of these gases [1-4]. Moreover this chemical warfare agents that include blood, vesicant, nerve, choking and blister agents in lower concentrations may approach the levels (removing oxygen and nitrogen from the space) are not geared for the atmosphere.

Therefore it is dramatically important to develop integrated smart wearable sensors which will improve the safety and efficiency of emergency personnel by monitoring the health status of the operator and the surrounding environment for potential risk sources. For these reasons field-effect capacitive EFIS (solid electrolyte-ferroelectric-insulator-semiconductor) and GSFET's (Gas-sensitive field-effect transistors) sensors with $\mathrm{BiFe}_{1-x} \mathrm{Mn}_{\mathrm{x}} \mathrm{O}_{3}$ sensing films are attractive, since miniaturization as well as integration with other types of sensors is feasible. They are appropriate for continuous controlling composition of hazardous gases: for a threat detection and identification within enclosed spaces. These sensors are important for environmental monitoring, industry and human life: air conditioning, food packaging, agriculture, biological technology and medical services. The application of doping materials to the sensing $\mathrm{BiFeO}_{3}$ film makes them essentially more thermally, chemically and physically stable than other materials.

Gas sensitivity of oxides is a very complicated phenomenon, which depends on grain size, porosity, grain faceting, bulk conductivity, surface architecture and stoichiometry, catalytic reactivity, band gap etc. $[4,5]$. The sensitivity of semiconductor oxide is associated with the easy exchange of electrons between adsorbate and gas sensitive material in both directions. Bismuth ferrites contain two metals: $\mathrm{Bi}$ and Fe which achieve two relatively easily accessible oxidation states.

A variety of methods is known (furnace, wet chemical, solgel synthesis, physical processing techniques, etc.) that can now be used to produce the ceramic materials, but they are both energyintensive and time-consuming or produce a variety of undesirable by-products. Among this technology the conventional ceramic technique is most applied, but it has some obvious disadvantages. It is difficult to prepare high purity $\mathrm{BiFe}_{1-\mathrm{x}} \mathrm{Mn}_{\mathrm{x}} \mathrm{O}_{3}$ ceramic material with homogeneous distribution of component and pores, with given or desired stoichiometrics of components $[6,7]$. 
In this work the general laws and characteristics of Self-Propagating High-Temperature Synthesis (SHS) for fabrication of $\mathrm{BiFeO}_{3}$ are studied which enable a more cost effective production than existing commercial processes. Among important advantages of SHS are simplicity of the process, high reaction rates, and no need for external energy [8]. Since the duration of SHS process is very short (few seconds) so the vaporization of bismuth oxide is negligible. The SHS process is characterised mainly by combustion temperatures and propagation velocity of the combustion front which are investigated.

\section{Experimental Procedure}

SHS is a kind of combustion in which valuable solid materials are formed via propagation of chemical reaction wave. SHS presents strong exothermal reaction (combustion) in which emerged heat is located in narrow layer and propagates from layer to layer by thermal conductivity and transforms the reactants into products (Fig.1). Hours is needed for synthesis of $\mathrm{BiFe}_{1-x} \mathrm{Mn}_{\mathrm{x}} \mathrm{O}_{3}$ materials by traditional furnace method while in a wave of combustion this problem is solved instantly and there is no necessity of complicated expensive equipment and a lot of energy consumption. By changing conditions of synthesis it is possible to regulate composition of gotten materials, microstructure, properties etc.

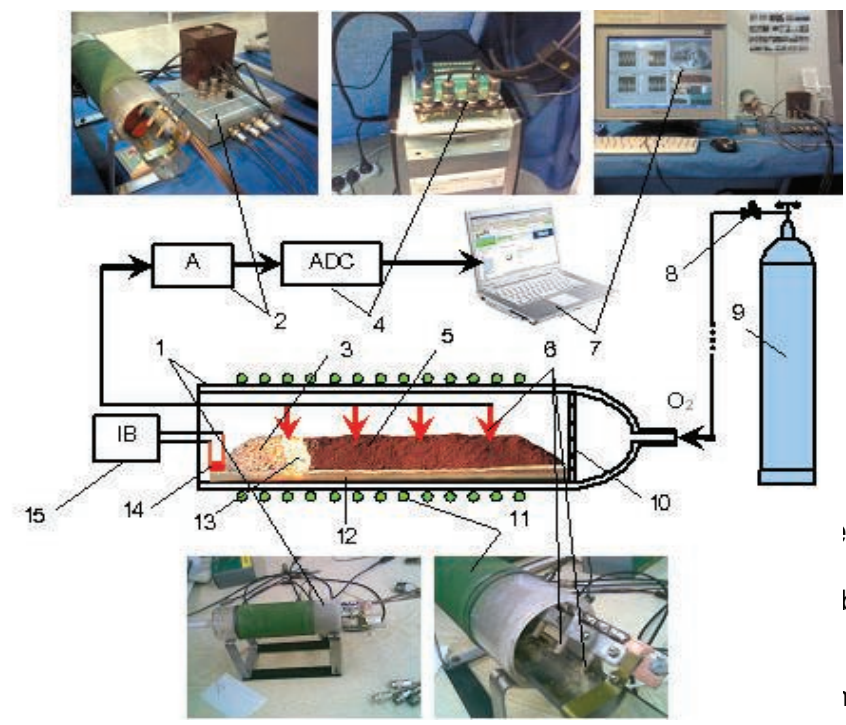

: schematics of laboratory SHS reactor.

be 2. Low noise amplifier 3. End product 4. Analog-digital Green mixture; 6. Thermocouples 7. PC 8. Oxygen flow Oxygen 10. Quartz mesh 11. Heater 12. Thermoresistant nbustion front 14. Wolfram ignitor 15. Ignition block.

The specificity of SHS requires the presence of fuel and oxidant in the initial mixture to create the combustion. $\mathrm{Fe}$ (iron powder) which is included in $\mathrm{BiFe}_{1-\mathrm{x}} \mathrm{Mn}_{\mathrm{x}} \mathrm{O}_{3}$ complex oxide composition is applied as fuel. Oxygen is used as an oxidant. Complex oxide $\mathrm{BiFe}_{1-x} \mathrm{Mn}_{\mathrm{x}} \mathrm{O}_{3}$ is synthesized from powder mixtures of Bismuth oxide $\mathrm{Bi}_{2} \mathrm{O}_{3}(99.975 \%)$, Iron oxide $\mathrm{Fe}_{2} \mathrm{O}_{3}(99.5 \%)$, Manganese dioxide $\mathrm{MnO}_{2}$ $(99.9 \%)$ and Iron $\mathrm{Fe}(99.9 \%)$. The syntheses are done according to the following chemical schemes:

$$
0.5 \mathrm{Bi}_{2} \mathrm{O}_{3}+(1-x)\left(k \mathrm{Fe}+\frac{(1-k)}{2} \mathrm{Fe}_{2} \mathrm{O}_{3}\right)+x \mathrm{MnO}_{2} \stackrel{\mathrm{O}_{2}}{\longrightarrow} \mathrm{BiFe}_{1-x} \mathrm{Mn}_{x} \mathrm{O}_{3} \text {, }
$$

where $\mathrm{k}$ is a factor controlling the exothermicity of the mixture, $\mathrm{x}$ is amount of acceptor dopants, in this work $\mathrm{x}$ was taken 0.3 .

The reactant particle size is known to have a strong impact on the combustion temperature and velocity. Commercially available reactant powders with average particle size smaller than $1 \mu \mathrm{m}$ are used in our experiments.

The general parameters of the SHS process (temperature and propagation velocity of the combustion front) are measured (Fig.2). Temperature is measured by C-type (tungsten) or B-type (Pt30\%RhPt6\%Rh) $0.1 \mathrm{~mm}$ diameter thermocouple placed in the center of pellets. The velocity of the 
combustion front is determined from the time needed for the front to move between two thermocouples in the sample. Automated data recording is done with the aid of LabView software.

Experiments have shown that the threshold value for $\mathrm{k}$ is 0.5 , only at greater values of $\mathrm{k}$ the process can proceed under the self-sustaining mode. The greater the value of combustible $(k)$ the higher the combustion temperatures and velocity. It should be noted also that high combustion velocities provide high output rates of the product from the reactor but undesirable processes of sintering and agglomeration increase. Influence of the amount of combustible in green mixture on degree of conversion of the initial charge to the final product was investigated. For it the series of powder were synthesized in different regimes and characterized by X-ray diffraction (XRD) at room temperature (Siemens D5000), using CuKa radiation, in the $20^{\circ}<2 \theta<60^{\circ}$ range with $0.008^{\circ}$ step size and an acquisition time per step of $10.6 \mathrm{~s}$. (Fig.3). And maximum quantity of a required phase is formed when the content of metal powder in the charge is in limits 10-12 wt. \% (Fig.3).

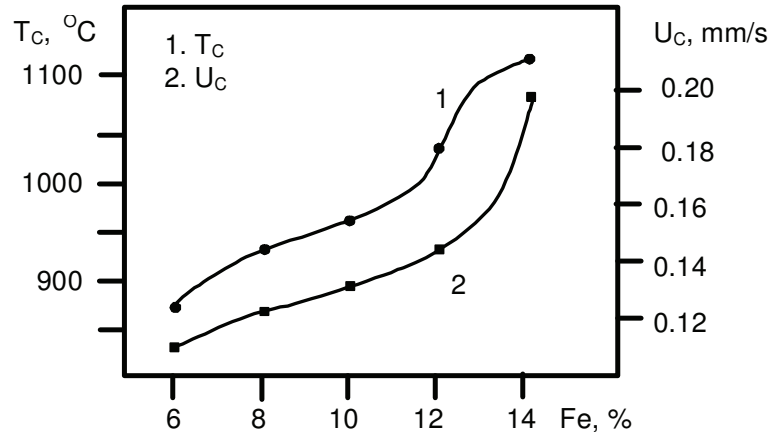

Fig.2. Combustion temperature (Tc) and velocity (Uc) vs. amount of combustible in the initial mixture.

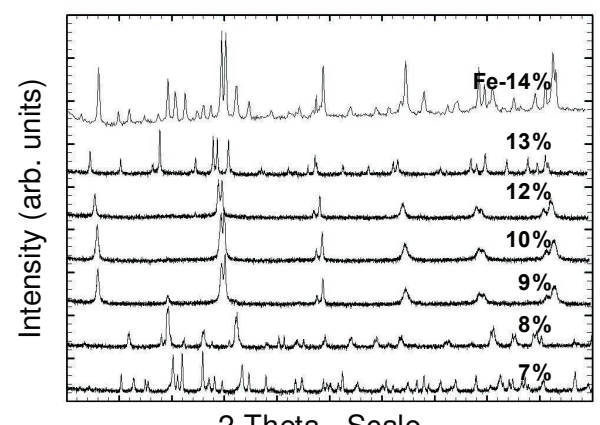

Fig.3. Relative intensity of phases $\left(1 / /_{0}\right)$ vs. amount of the combustible (Fe, \%).

It is observed that the use of initial reagents from various manufacturers may result in different densities of the initial mixtures, thus influencing the operating regimes of the SHS reactor. The investigations of the impact of relative densities of the initial mixtures on the combustion temperatures and velocity shows that the increase in relative density of the initial charge leads to the decreased combustion temperature and velocity, caused by difficulty of diffusive access of oxygen to the combustion zone. At the relative densities ( $\rho_{\text {pressed }} / \rho_{\text {poured }}$ ) more than 1.6 the SHS process is interrupted because of deficit of oxygen. XRD data have shown that the degree of conversion of the initial charge into the final product has inverse dependence on the initial charge density. Both the amounts of the combustible and density of the initial charge affect the conversion degree through the combustion temperatures and front propagation velocity.

The synthesized compositions are grounded by planetary agate boil mill with acetone as a milling media until to obtain particle sizes of 5 to $8 \mu \mathrm{m}$. $0.1 \mathrm{~mol}$. Bi is added to create $\mathrm{Bi}_{1.1} \mathrm{FeO}_{3}$ ceramic with $\mathrm{Bi}$ excess as a target to compensate for the $\mathrm{Bi}$ volatilization during pulsed laser deposition. 10\% solution of polyvinyl alcohol was added in the milled powders to form a paste which is pressed into disks 6-14 $\mathrm{mm}$ in diameter and 6-8 $\mathrm{mm}$ thick under different pressure. Next the kinetics of sintering for samples are studied: $\mathrm{BiFe}_{1-x} \mathrm{Mn}_{\mathrm{x}} \mathrm{O}_{3}$ based pellets are then sintered between $700{ }^{\circ} \mathrm{C}$ and $850{ }^{\circ} \mathrm{C}$ in an electric furnace and air atmosphere under controlled heating/cooling rates of $2.5^{\circ} \mathrm{C} / \mathrm{min}$ with $2 h$ dwell time. Dependence for linear shrinkage factor and gas-penetrability of samples vs. sintering temperature has indicated that intensive sintering begins with $740^{\circ} \mathrm{C}$ (Fig.4), and maximum density of samples is practically obtained at $760^{\circ} \mathrm{C}$ (it's important that the main phase is $\mathrm{BiFeO}_{3}$, as shown in Fig.5). The conditions in which samples were prepared also act upon the sintering kinetics. For this purpose samples were pressed at different pressures (pressing pressure was changed from 1000 $\mathrm{kg} / \mathrm{cm}^{2}$ to $5000 \mathrm{~kg} / \mathrm{cm}^{2}$, accordingly with different densities) and were subjected to simultaneous sintering at $760^{\circ} \mathrm{C}$, it is experimentally found that optimal pressure of pressing to decrease the linear shrinkage factor and grow the density of ceramic samples is about $4000 \mathrm{~kg} / \mathrm{cm}^{2}$. 


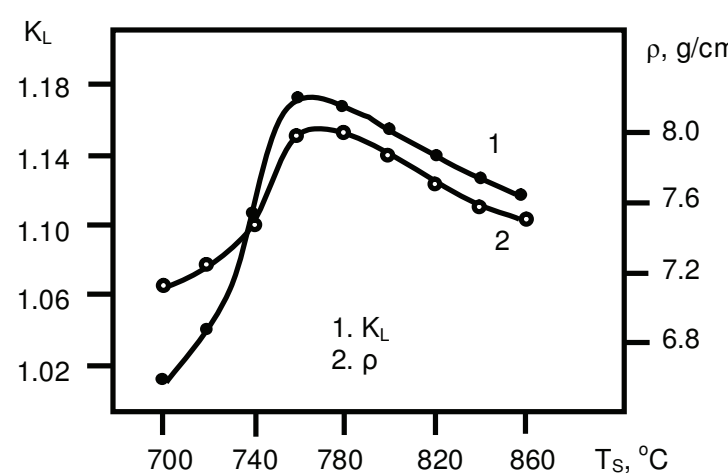

Fig.4. Linear shrinkage factor and density of ceramic samples vs. sintering temperature.

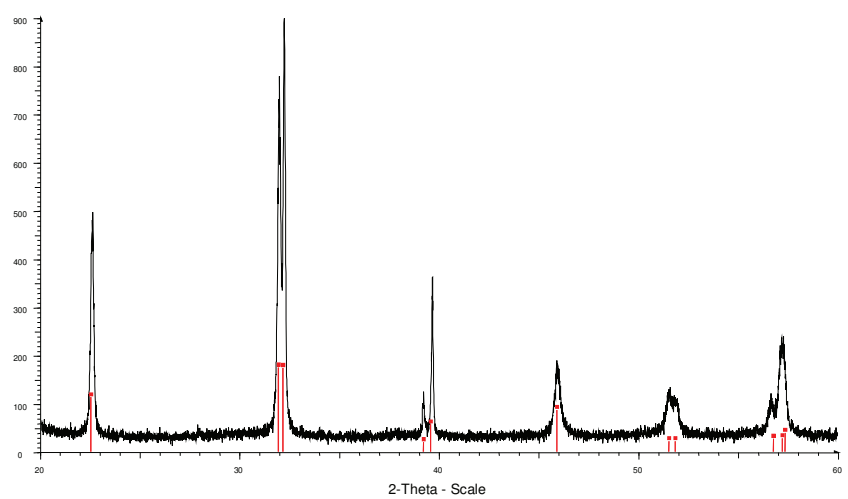

Fig.5. X-ray diffraction pattern of $\mathrm{BiFe}_{1-\mathrm{x}} \mathrm{Mn}_{\mathrm{x}} \mathrm{O}_{3}$ ceramics sintered at $760{ }^{\circ} \mathrm{C}$ for 2 hour.

Possibilities of making contactless sensors by monitoring the dielectric/magnetic properties using microwave cavities are considered. For this purpose gas sensors based on a field-effect capacitive structure with thin-film $\mathrm{BiFe}_{1-x} \mathrm{Mn}_{\mathrm{x}} \mathrm{O}_{3}$ perovskite (Fig.6) are prepared by pulsed laser deposition (PLD) technique and investigated. The Au/TiO2 (500/50 nm thick) IDC electrodes are deposited by magnetron sputtering on fused silica substrates and subsequently patterned by $\mathrm{Ar}$ ion milling. The BFO films (700 nm thick) are grown by PLD using a $\operatorname{KrF}$ excimer laser $(\lambda=248 \mathrm{~nm}, \mathrm{~T}=30 \mathrm{~ns})$ operating at $10 \mathrm{~Hz}$ with an energy density of $1.5 \mathrm{~J} \cdot \mathrm{cm}^{-2}$. The oxygen pressure during deposition was maintained at 0.01 mbar. A series of samples prepared at different BFO film growth temperatures (varying in the range $550-650{ }^{\circ} \mathrm{C}$ ) is fabricated (Fig. 7).

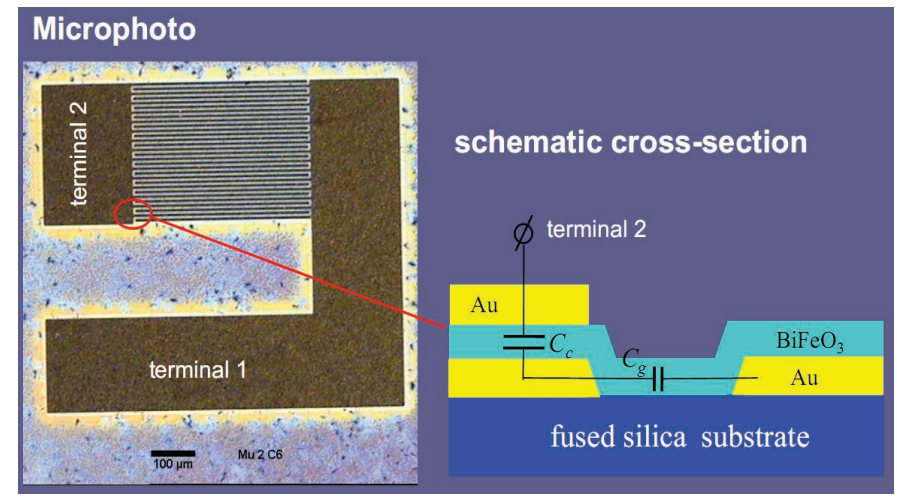

Fig. 6. Inter-digital capacitor Structure of Sensor.

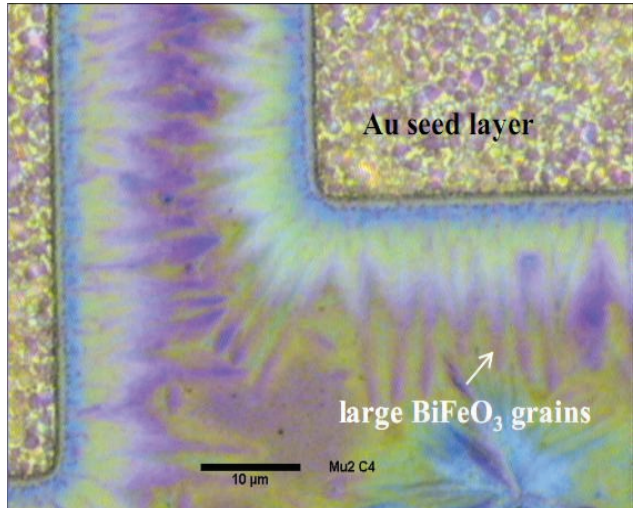

Fig. 7. In plane grain growth.

\section{Results and discussion}

Silver or gold ohmic contacts were deposited on ceramic thin films. The main electro-physical, conventional-sensorial properties and mechanical characteristics of prepared ceramic samples were investigated. The electrical resistance measurements of gas-sensitive elements were carried out under various concentrations of acetone, ethanol, natural gas and petrol in the temperature range 250 to $450{ }^{\circ} \mathrm{C}$. The gas sensitivity as a function of temperature is shown in Fig. 8 and as a function of the concentration of analyzed gas in the air present in the test chamber is shown in Fig.9.

For electrical characterizations of field-effect capacitive structure the $\mathrm{Au} / \mathrm{Ti}(500 / 50 \mathrm{~nm}$ thick) pads are deposited by e-beam evaporation on top of BFO films and patterned by lift-off process. The pads are capacitively connected (using BFO as dielectric) to the bottom contact pads of the IDC structure [9]. 


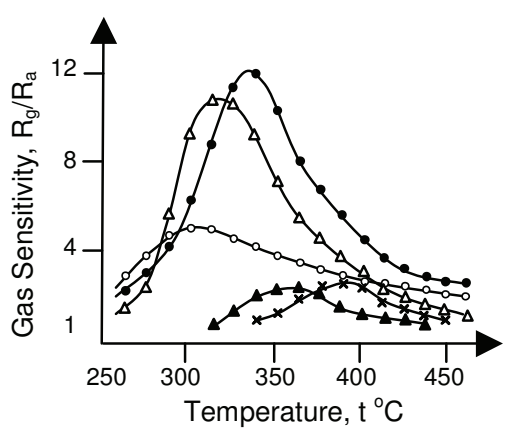

Fig.8. Gas sensitivity vs. temperature for ceramic samples: -acetone 0,1 vol.\%; $\Delta$-ethanol 0,07 vol.\%; o-natural gas 1 vol.\%; $\boldsymbol{\Delta}$ - petrol 0,05 vol. $\%$; $x$-acetone 0,1 vol. $\%$ in argon.

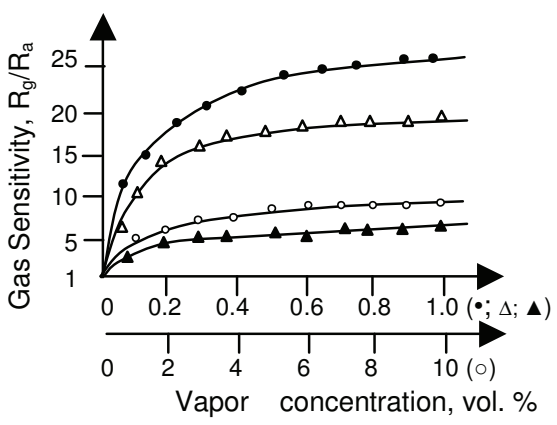

Fig.9. Gas sensitivity of $\mathrm{BiFeO} 3$ ceramic samples a function of the concentration of various vapors in air: -acetone at $340{ }^{\circ} \mathrm{C} ; \Delta$-ethanol at $320^{\circ} \mathrm{C}$; o-natural gas at $290^{\circ} \mathrm{C}$; $\boldsymbol{\Delta}$ - petrol at $390^{\circ} \mathrm{C}$.

The dielectric response of the test structures is measured using a HP 4285A LCR-meter at $1 \mathrm{MHz}$ and an Agilent N5230 vector network analyzer in the frequency range $0.01-45 \mathrm{GHz}$. A bias tee is used to apply up to $150 \mathrm{~V}$ dc bias. The permittivity of BFO films is calculated using Farnell's analysis [10]. The dependence of capacitance of an IDC structure on electric field is shown in Figure 10. Figure 11 shows permittivity and loss tangent of IDC structures versus frequency. The increase of permittivity and loss tangent at higher frequencies is due to self resonance of the IDC structure including the BFO film. The apparent permittivity, 24, is in very good agreement with the bulk value [11, 12]. At $1 \mathrm{GHz}$ the loss tangent is less than 0.02 which is rather low and acceptable for microwave applications.

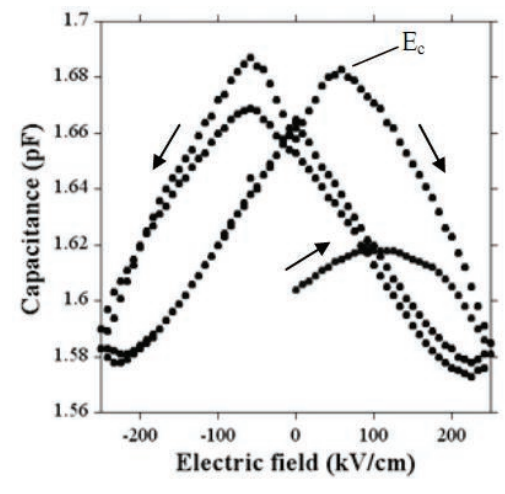

Fig.10. Capacitance of BFO test structures versus electric field: high field measurements.

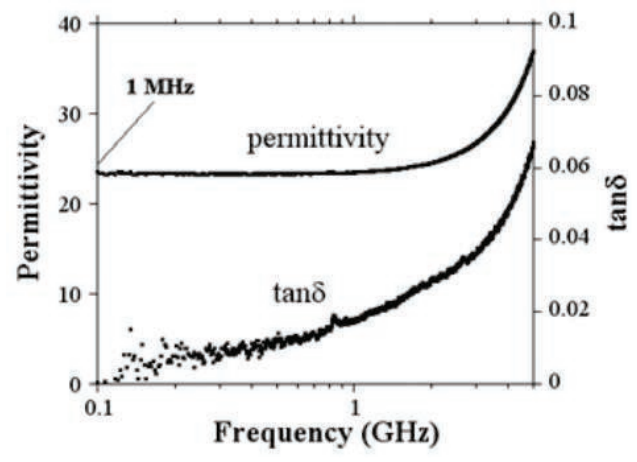

Fig.11. Permittivity and loss tangent of a BFO test structure versus frequency.

The gas-sensitive properties of field-effect sensors were tested in temperature range from $250{ }^{\circ} \mathrm{C}$ to $400{ }^{\circ} \mathrm{C}$ in 0.2 vol. \% concentrations of acetone, ethanol, petrol and 2 vol.\% concentration of natural gas at a frequency of $1 \mathrm{MHz}$, the sensors with BFO films show an average gas sensitivity of $\varepsilon_{a} / \varepsilon_{g}=1.3$; $1.25 ; 1.4 ; 1.2$ respectively.

\section{Conclusions}

The experiments of SHS have shown that the values of combustion temperature and front propagation velocities are $800-1100{ }^{\circ} \mathrm{C}$ and $0.1-0.2 \mathrm{~mm} / \mathrm{s}$, respectively, when the content of combustible was changed from $6 \%$ to $14 \%$. The phase structures were studied by X-ray diffractometer (XRD) and found out that best results were gotten when amount of combustible (Fe powder) is in the range of 10$12 \%$. The gas sensitivity as a function of temperature (up to $450{ }^{\circ} \mathrm{C}$ ) and the concentration (up to 10 vol.\%) of analyzed gas in the air present in the test chamber is investigated, the sensors show high sensitivity at temperature of $250-400{ }^{\circ} \mathrm{C}$. The permittivity 24 and coercive field $60 \mathrm{kV} / \mathrm{cm}$ of the proposed IDC structures with large BFO grain films are like in the bulk counterpart. Loss tangent is 
less than 0.02 at $1 \mathrm{GHz}$ which is acceptable for microwave applications. The field-effect capacitive sensors with BFO films show an average gas sensitivity of $\varepsilon_{\mathrm{a}} / \varepsilon_{\mathrm{g}}=1.2 \div 1.4$.

\section{Acknowledgments}

I'm grateful to Dr. S. Gevorgian and Dr. A. Vorobiev (Department of Microtechnology and Nanoscience, Chalmers University of Technology, SE-41296 Gothenburg, Sweden) for their assistance during this study.

\section{References}

1. R.R. Saraf, S.S.Thipse and P.K.Saxena. Comparative Emission Analysis of Gasoline/LPG Automotive Bifuel Engine. International Journal of Civil and Environmental Engineering 1:4 2009.

2. Jiann-Hwa Liou, Po-Jou Liou, Tzer-Shin Sheu, "Physical Properties and Crystal Chemistry of Bismuth Oxide Solid Solution," Processing and Characterization of Electrochemical Materials and Devices. Proc. Symp. Indianapolis, 25-28 April 1999, pp 3-10. Ceram. Trans. 109.

3. Y. K. Jain and V. K. Khanna. Thick Film, LTCC or Silicon Microhotplate for Gas Sensor and Other Applications. Int. J. Appl. Ceram. Technol.

4. M.L. Post, J.J. Tunney, D. Yang, X. Du, D.L. Singleton. Material chemistry of perovskite compounds as chemical sensors. Sensors and Actuators B: Chemical, Volume 59, Number 2, 19 October 1999, pp. 190-194(5).

5. M. A. Peña, J. L. G. Fierro. Chemical Structures and Performance of Perovskite Oxides. Chem. Rev. 2001, 101, 1981-2017.

6. Meiya Li, Min Ning, Yungui Ma, Qibin Wu andCKOng. Room temperature ferroelectric, ferromagnetic and magnetoelectricproperties of Ba-doped BiFeO3 thin filmsJ. Phys. D: Appl. Phys. 40 (2007) 1603-1607.

7. Sverre M. Selbach, Mari-Ann Einarsrud, Thomas Tybell, and Tor Grande. Synthesis of BiFeO3 by Wet Chemical Methods. J. Am. Ceram. Soc.,1-5 (2007).

8. Merzhanov A.G., Self-propagating high-temperature synthesis: Twenty years of search and findings. In: Combustion and Plasma Synthesis of High-Temperature Materials/ Eds. Z.A. Munir, J.B. Holt. N.Y.: VCH Publ., 1990, p.1-53.

9. A. Vorobiev, N. Martirosyan, S.G. Eriksson and S. Gevorgian. Growth of dielectric characterization of large grain $\mathrm{BiFeO} 3$ thin films on amorphous $\mathrm{SiO} 2$ substrates. IOP Conf. Series: Materials Science and Engineering 8 (2010) 012001.

10. Al-Shareef H N, Dimos D, Raymond M V, Schwartz R W and Mueller C H 1997 J. Electroceram. 1145.

11. Roginskaya Yu E, Tomashpol'skii Yu Ya, Venevtsev Yu N, Petrov V M and Zhdanov G S. 1966, Zh. Eksp. Teor. Fiz. 50 69; 1966 Sov. Phys. JETP (English Transl.) 2347.

12. Kamba S, Nuzhnyy D, Savinov M, Sebek J, Petzelt J, Prokleska J, Haumont R and Kreisel J. 2007 Phys. Rev. B, 75024403. 